Review Article

\title{
Cardiovascular Manifestations of SARS CoV-2: A Review
}

\author{
Naresh Kumar', $\underline{\text { Satish Kumar }}{ }^{2}$, Harsh Vardhan $^{3}$, Lovika Lakhtakia ${ }^{4}$, MK Daga $^{5}$ \\ ${ }^{1}$ Professor of Medicine and Head, Pulmonary Medicine, Maulana Azad Medical College, New Delhi, India. \\ ${ }^{2}$ Senior Resident, Department of Medicine, King George's Medical University, Lucknow, U.P., India. \\ ${ }^{3,4}$ Senior Resident, Department of Pulmonary Medicine, Maulana Azad Medical College, New Delhi, india. \\ ${ }^{5}$ Director Professor, Department of Medicine, Maulana Azad Medical College, New Delhi, india. \\ DOI: https://doi.org/10.24321/2349.7181.202005
}

I $\quad \begin{array}{lllll}\mathbf{N} & \mathbf{F} & \mathbf{O}\end{array}$

Corresponding Author:

Naresh Kumar, Professor of Medicine and Head, Pulmonary Medicine, Maulana Azad Medical College, New Delhi, India.

E-mail Id:

drnareshmamc@gmail.com

Orcid Id:

https://orcid.org/0000-0003-4581-609X

How to cite this article:

Kumar N, Kumar S, Vardhan H, Lakhtakia L, Daga MK. Cardiovascular Manifestations of SARS CoV2: A Review. J Adv Res Med 2020; 7(2): 1-10.

Date of Submission: 2020-06-10

Date of Revision: 2020-07-30

Date of Acceptance: 2020-09-05
$\begin{array}{llllllll}\mathbf{A} & \mathbf{B} & \mathbf{S} & \mathbf{T} & \mathbf{R} & \mathbf{A} & \mathbf{C} & \mathbf{T}\end{array}$

Severe acute respiratory syndrome coronavirus 2 (SARS-CoV-2) pandemic is a public health emergency of global concern. Besides the profound severe pulmonary damage, SARS-CoV- 2 infection also causes a series of cardiovascular abnormalities, including myocardial injury, myocarditis and pericarditis, arrhythmia and cardiac arrest, cardiomyopathy, heart failure, cardiogenic shock, and coagulation abnormalities. COVID-19 patients with preexisting cardiovascular diseases are often at a much higher risk of increased morbidity and mortality. Number of mechanisms have been postulated for SARS CoV-2-associated cardiovascular damage including direct myocardial injury, systemic inflammation, hypoxemia, coronary thrombosis and drug-induced cardiac damage. Special attention should be given towards patients of SARS CoV- 2 with concurrent cardiovascular diseases. Knowledge of the injury caused by SARS CoV-2 to the cardiovascular system and the mechanisms behind it is of the utmost importance to reduce the morbidity and mortality of these patients by treating them in time.

Keywords: SARS CoV-2, COVID 19, Cardiovascular Manifestation, Myocardial Injury, Myocarditis, Arrhythmia

\section{Introduction}

An outbreak of pneumonia of unknown cause was detected in Wuhan, Hubei province in December 2019. ${ }^{1}$ These pneumonia patients underwent testing and gene sequencing leading to identification of the pathogen named as 2019 novel coronavirus (2019-nCoV), which was further named officially as severe acute respiratory syndrome coronavirus 2 (SARS-CoV-2) by the World Health Organisation (WHO). ${ }^{2}$ SARS-CoV-2 has spread rapidly across China and became pandemic. ${ }^{1}$ Symptomatic patients usually presented with fever, dry cough, shortness of breath, myalgia or fatigue. The median incubation period in COVID-19 is 5.1 days with majority of them $(97.5 \%)$ becoming symptomatic within
11.5 days of infection. ${ }^{2}$ In the current scenario, the statistics of SARS-CoV- 2 are staggering and relentlessly mounting. It is very clear that the number of infections and deaths are continuously increasing with increasing need for critical care interventions. The clinical spectrum of SARS-CoV-2 ranges from mild to severe with a majority of them (81\%) having mild symptoms while severe symptoms (defined as respiratory rate $\geq 30 / \mathrm{min}$, hypoxia: blood oxygen saturation $\leq 90 \%, \mathrm{PaO} 2 / \mathrm{FiO} 2<300$, and/or pulmonary infiltrates $>50 \%$ within 24 to $48 \mathrm{~h}$ ) occur in $14 \%$ cases. Critical illness (defined as respiratory failure, septic shock, and/or multiple organ dysfunction or failure) have been reported in only $5 \%$ of subjects with overall Case Fatality Rate (CFR) of 
2.3\%. ${ }^{3}$ studies have suggested that older population and persons with chronic health problems like Cardiovascular Disease (CVD) are at higher risk for COVID related morbidity and morbidity than the average population. ${ }^{4,5}$ However, severe disease requiring hospital admissions and even expiries have been observed in younger individuals as well. Awareness about natural history of COVID-19 is increasing day by day but data regarding cardiovascular involvement are scarce. Usually respiratory symptoms dominate as clinical manifestations of COVID-19, but some of the cases have severe cardiovascular damage as well. ${ }^{6}$ In addition, the presence of cardiovascular co-morbidities such as hypertension, coronary artery disease in these patients are often associated with poor prognosis. ${ }^{3}$ So, knowledge of the injury caused by SARS-CoV-2 to the cardiovascular system and the mechanisms behind this is of utmost importance to reduce the mortality by treating these conditions in time.

\section{Pathophysiology of Cardiac Manifestations}

The mechanisms of cardiovascular damage from SARS-CoV-2 have not been fully understood however, it seems to be due to multiple factors. The common pathological mechanisms involved in causing cardiovascular complications are: direct myocardial injury, systemic inflammation, hypoxia induced injury and rupture of plaque and coronary thrombosis.

\section{Direct Myocardial Injury}

Angiotensin-Converting Enzyme 2 (ACE2) is a membranebound aminopeptidase that plays an important role in cardiovascular and immune systems. ${ }^{7}$ ACE2 has a major role in cardiac functioning and the development of high blood pressure and diabetes mellitus. ACE2 has been found as a functional receptor for SARS-CoV and SARS-CoV-2. ${ }^{7}$ SARS-CoV-2 infection is caused following binding of viral spike protein to human Angiotensin-Converting Enzyme 2 (ACE2) receptor which is expressed in the lungs (on type Il pneumocytes), oral mucosa, heart, intestine, kidneys and vascular endothelium. Organ distribution of ACE2 seems to be closely related to the clinical involvement. The binding of SARS-CoV-2 to ACE 2 results in alteration in ACE2 signaling pathways that leads to acute myocardial injury as well as lung injury. Binding of SARS-CoV-2 to ACE2 prevents the enzyme from converting Ang II to Ang 1-7, potentiating Ang II-induced biological effects including pro-inflammation, pro-fibrosis, pro-oxidation, and vasoconstriction leading to cardiovascular and lung injury in the face of SARS-CoV-2 infection. ${ }^{8,9}$ Ang 1-7 offers an array of benefits including ant inflammation, anti-fibrosis, anti-oxidation, and vasodilatation. ${ }^{8}$

\section{Systemic Inflammation}

Severe form of infection with SARS-CoV-2 is characterized by acute systemic inflammatory response and cytokine storm which results in immune mediated injury to various organs leading to multiorgan dysfunction. Cytokine storm syndrome denotes a severe life-threatening condition manifested by overwhelming inflammation, a sharp rise in proinflammatory cytokines, acute phase reactants like IL-6, hyperferritinemia, CRP, hemodynamic instability, and multi-organ failure, and is potentially fatal if untreated. ${ }^{10}$ The hallmark of cytokine storm is an uncontrolled and dysregulated immune response involving continued activation of lymphocytes, macrophages, and natural killer cells. ${ }^{11}$ The viral RNA serves as the main pathogenic molecule which binds to the pattern recognition receptors such as Toll-Like Receptor (TLR) 3, TLR7, TLR8 and TLR9. This leads to triggering of the downstream inflammatory cascade and activation of nuclear factor-KB (NF-KB) and Interferon Regulatory Factor 3 (IRF3) commencing in production of IFN- $\alpha / \beta$ and other pro-inflammatory molecules such as IL-1, IL-2, IL-4, IL-6, IL-10, IL-12, GCSF, IFN- $\gamma$ and TNF- $\alpha$ amongst which IL- 6 is the most important one. This massive production of cytokines leads to increased vascular permeability, alveolar epithelial damage, ARDS followed by multisystem involvement. ${ }^{12}$ The syndrome mainly impairs the cardiovascular system and manifests as severe heart failure and cardiogenic shock, accompanied with extracardiovascular symptoms including fever, lymphadenitis, rashes on hands and feet, and stomach ache. $^{13}$

\section{Hypoxemia}

There is already a state of increased cardiometabolic demand in heart due to systemic inflammation. Hypoxia further alters myocardial demand-supply ratio causing acute myocardial injury, arrhythmia and shock. In addition, influx of calcium ions can be induced by hypoxemia and cause apoptosis and injury to cardiomyocytes. ${ }^{14}$

\section{Coronary Thrombosis and Plaque Rupture}

Coronary artery disease of Img duration along with the risk factors of atherosclerotic disease may enhance the occurrence of acute coronary syndrome after an infection as already shown in epidemiologic and clinical studies of influenza virus and other acute inflammatory conditions. ${ }^{15-17}$ Infection triggers an increase in myocardial oxygen demand, which precipitates myocardial injury and infarction. Hypoxia too has been postulated as one of the causes of hypercoagulable states in these patients. In the same sequence, circulating cytokines released at the time of severe systemic inflammatory response may cause the breaking of atherosclerotic plaque and its instability. Proinflammatory cytokines may itself lead to endothelial injury and activation of coagulation cascade. ${ }^{18}$ In such scenario, levels of D-dimer which serves as marker of fibrinolytic activity is elevated along with other inflammatory cytokines. Individuals with cardiac disorders are also at risk for hemodynamic decompensation at the time of stress due 
to severe infectious illnesses. Critically ill patients with COVID-19 are at an increased risk for venous thrombosis and hence the need for anticoagulation in these patients is justified.

\section{Drug-Induced Cardiovascular Toxicity}

At this point, antiviral drug-induced cardiovascular toxicity in SARS-CoV-2 treatment should not be ignored. Antiviral drugs including IFN- $\alpha$, ribavirin, chloroquine phosphate, lopinavir/ritonavir and remdesivir have all been included in the treatment of COVID-19. ${ }^{19}$ Several antiviral drugs exert cardiotoxicity or elicit interactions with other cardiac medications. For instance, lopinavir/ ritonavir may lead to prolongation of PR and QT intervals and influence serum levels of antiplatelet drugs through CYP3A4 inhibition. ${ }^{20}$ Hydroxychloroquine (HCQ) mainly acts by (a) inhibiting ACE2-mediated viral entry hence preventing SARS CoV- 2 infection and (b) anti-inflammatory and immunomodulatory property hence attenuating the cytokine storm. However, due to its inherent property of blocking the hERG/Kv11.1 potassium channel, it can prolong the QT interval and increase risk of Torsades de pointes (TdP) especially in patients with congenital long QT syndrome. ${ }^{20}$ This risk further gets compounded when drugs like azithromycin or lopinavir and ritonavir are prescribed simultaneously with HCQs.

\section{Other Possible Mechanisms}

Any systemic illness when critical can cause electrolyte imbalances which can precipitate arrhythmias especially in patients with preexisting cardiac disorders. Hypokalemia, in particular, resulting from the interaction of SARS-CoV-2 with renin angiotensin system (RAS) increases the vulnerability of heart to various tachyarrhythmias.

\section{COVID- 19 and Cardiovascular Manifestations}

Studies available so far depicts close relationship between cardiovascular disease and SARS or MERS. ${ }^{4}$ Patients of SARS-CoV infection experiences a variety of cardiovascular complications like tachycardia (71.9\%), hypotension (50.4\%), bradycardia (14.9\%), reversible cardiomegaly (10.7\%) and transient atrial fibrillation. ${ }^{8}$ Elevation of cardiac biomarkers, arterial and Venous Thromboembolism (VTE), cardiogenic shock and arrest are the other cardiovascular manifestations of COVID-19. An observational study on Covid-19 patients has shown complications like shock (8.7\%), acute cardiac injury (7.2\%), arrhythmias (16.7\%), acute respiratory distress syndrome (ARDS) (19.6\%), and acute kidney injury (3.6\%). ${ }^{21}$ Particular forms of cardiovascular complications or aggravation of preexisting cardiovascular conditions in COVID-19 patients are discussed in detail here.

\section{Acute Myocardial Injury}

Myocardial injury, characterized by elevated levels of cardiac biomarkers, results from myocardial ischemia and nonischemic causes including myocarditis. ${ }^{22}$ Several studies have noted acute myocardial injury in patients with SARS CoV-2 which mainly manifested as follows: (1) cardiac biomarkers (hypersensitive cardiac troponin I) > 99th percentile upper reference limit; or (2) new abnormalities on electrocardiogram or echocardiogram..$^{23}$ In a series of 41 patients from Wuhan, China evidence of myocardial injury was seen in $12 \%$ (5) with majority of them 4 (80\%) requiring ICU care. ${ }^{24}$ Similarly, Wang et al. documented evidence of acute cardiac injury in $7.2 \%(10 / 138)$ patients of SARS CoV-2, of whom $8(80 \%)$ required an ICU care. ${ }^{25} \mathrm{~A}$ higher utilization of ICU care among these patients with myocardial injury suggests a more severe and advanced disease process in them. In a meta-analysis of 341 patients from China (four studies), it was seen that cardiac troponin I levels were significantly higher in patients with severe disease (standardized mean difference $25.6 \mathrm{ng} / \mathrm{l} ; 95 \% \mathrm{Cl}$ [6.844.5]). The authors postulated that an early measurement of cardiac troponins immediately post admission would help identify a subset of patients with cardiac injury thus portraying a worse outcome. ${ }^{26}$

The mechanism for myocardial injury include virus mediated direct myocardial injury, immune mediated myocardial injury (systemic inflammation) and hypoxemia, as elaborated in pathophysiology. ${ }^{27}$ An autopsy-based case report documented few mononuclear inflammatory infiltrates in myocardial interstitium on cardiac biopsy in a patient who succumbed to SARS- CoV-2. ${ }^{28}$ Another report has documented the presence of low grade myocardial inflammation and viral particles in the myocardium (electron microscopy) on endomyocardial biopsy in a patient of SARS CoV-2 with cardiogenic shock. ${ }^{29}$ Cytokine release syndrome or cytokine storm, a weakly known immunopathological condition due to hyperinduction of proinflammatory cytokines like interleukin (IL)-1, IL-6, T helper 1 cytokine interferon-gamma, and Tumour Necrosis Factor-alpha (TNF- $\alpha$ ), has been found in the mechanism of SARS, MERS, and influenza. ${ }^{30,31}$ In a retrospective series of 54 patients of SARS CoV-2, CRP levels were higher in patients with myocardial injury than those without indicating a severe inflammatory response in the former group. ${ }^{32}$ Thirdly, hypoxemia as a part of respiratory failure in these patients may lead to myocardial oxygen demand supply mismatch especially in those with preexisting cardiac disease culminating in myocardial damage.

Two articles from two different academic hospitals in Wuhan, China, which was the epicenter of SARS CoV2 favored the concept that inflammation is a potential mechanism for myocardial injury. ${ }^{25,33}$ Shi et al did a cohort study on 416 admitted cases with COVID-19 positivity by RT-PCR out of which 82 (19.7\%) had elevated high-sensitivity troponin I (TnI) levels, indicating myocardial injury with a 
higher mortality rate of $51.2 \%(42 / 82) .{ }^{34}$ Similar results were reported by Guo et al in 187 admitted patients of SARS CoV-2, of whom $27.8 \%$ (52) had increased levels of troponin T (TnT), and mortality of $59.6 \%(31 / 52) .{ }^{35}$ They reported that even if the functional and morphological cardiac dysfunctions were infrequent with elevated troponin values, it had a significant hazard ratio of 8.9 (95\% confidence interval 1.9-40.6) for the risk of expiry, as reported by others. ${ }^{25}$ Guo et al reported that TnT values are significantly linked with $C$-reactive protein and N-terminal pro-B-type natriuretic peptide (NT-proBNP) values thus associating myocardial injury to the grade of inflammation and ventricular dysfunction. They also showed that progressive serial increase in both $\mathrm{TnT}$ and NT-proBNP values are associated with worse outcome. ${ }^{35}$

\section{Acute Coronary Syndrome (ACS)}

As with other infectious diseases, including SARS and influenza, COVID-19 can trigger ACS. ${ }^{36,37}$ In early studies from China, a small proportion of patients with COVID-19 presented with chest pain on admission to hospital, but the characteristics of the chest pain were not described. ${ }^{4}$ In a case series from New York involving 18 patients with SARS CoV-2, and ST segment elevation indicative of potential acute myocardial infarction, five of the six patients with myocardial infarction required percutaneous coronary intervention. ${ }^{38}$ In a case series from Italy involving 28 patients of SARS CoV-2 and ST segment elevation myocardial infarction (STEMI), myocardial infarction was the first clinical manifestation of SARS CoV-2 in 24 patients and clinical assessment by coronary angiography showed that 17 patients had evidence of a culprit lesion that required revascularization. ${ }^{39}$ This suggests that SARS CoV-2 can cause ACS even in the absence of substantial systemic inflammation. However, the incidence of ACS in patients with SARS CoV-2 is still unknown. Considering the overwhelmed health-care facilities of many cities during the COVID-19 outbreak, the number of cases of acute myocardial infarction among patients with COVID-19 might be an underestimation in early studies. The underlying mechanism for SARS CoV-2 induced ACS might involve plaque rupture, coronary spasm or microthrombi in coronary vessels owing to systemic inflammation or cytokine storm..$^{40}$ Activated macrophages secrete collagenases that degrade collagen, a major constituent of the fibrous cap on atherosclerotic plaques, which can lead to plaque rupture. Activated macrophages are also known to secrete tissue factor, a potent procoagulant that triggers thrombus formation when the plaque ruptures. ${ }^{40}$ SARS CoV- 2 induced direct endothelial or vascular injury might also increase the risk of thrombus formation and subsequent ACS. ${ }^{41}$ Despite the potential for SARS CoV-2 to induce ACS, the number of reported cases of ACS during the outbreak in Italy, Spain and the USA was actually significantly lower than during pre-COVID-19 periods, with a reported $42-48 \%$ reduction in hospitalizations for ACS and a $38-40 \%$ reduction in percutaneous coronary interventions for STEMI. ${ }^{42}$ In contrast, the incidence of out-of-hospital cardiac arrest increased during this period, which was strongly associated with the cumulative incidence of COVID-19. ${ }^{43}$ This decline in number of patients with myocardial infarction seeking urgent hospital care during the peak of the outbreak was also reported in an extensive global survey by the ESC. ${ }^{43}$

\section{Myocarditis and Pericarditis}

Myocarditis can be one of the clinical presentations of SARS CoV-2 infection, however, its exact pathogenesis is still not clear. Viral infections like influenza and parvovirus B-19 are common causes of myocarditis. ${ }^{30,25}$ Viral myocarditis may present as either as focal or global myocardial inflammation, necrosis, and ventricular dysfunction. The data on the incidence of fulminant myocarditis in SARS CoV-2 is very much limited. Zeng et al. reported the first case of SARS CoV-2 presenting with fulminant myocarditis in a 63-year-old male with no underlying cardiac disease.${ }^{44}$ On admission, cardiac enzymes (troponin-I: $11.37 \mathrm{~g} / \mathrm{l}$, myoglobin: 390.97 $\mathrm{ng} / \mathrm{ml}$ ) and N-terminal brain natriuretic peptide (NT BNP: 22600 pg/ml) were elevated. ECG showed sinus tachycardia with no ST elevation. Echocardiography revealed enlarged cardiac chambers, dyskinetic myocardium with decreased LVEF (32\%). Similarly, Hu and colleagues reported a 37-yearold SARS CoV-2 positive male who presented with acute onset chest pain and hypotension with ST elevation in inferior leads and raised cardiac enzymes and BNP. ${ }^{45}$ CT coronary angiography revealed normal epicardial coronaries while echocardiography showed enlarged cardiac chambers, severely decreased ventricular function (LVEF: 27\%) with minimal pericardial effusion. He tested negative for other viruses causing myocarditis. Patient improved markedly with IV corticosteroids, immunoglobulins and inotropic support along with broad spectrum antibiotics (pre-discharge LVEF: $66 \%$ ). Most of the diagnoses of myocarditis have been made based on clinical and ECG findings, elevated cardiac enzymes, echocardiographic evidence with normal coronaries on coronary angiogram. ${ }^{46} \mathrm{In}$ an analysis of 68 fatal cases of SARS CoV-2, 5 patients (7\%) were found with fatal fulminant myocarditis in combination with circulatory failure, and 22 fatalities (33\%) were attributed to both myocarditis and respiratory failure. ${ }^{47}$ Direct localization of SARS CoV- 2 in myocardium and systemic inflammatory response may attribute to myocarditis in these patients.

\section{Arrhythmia and Cardiac Arrest}

Cardiac arrhythmias both tachy- as well as bradyarrhythmias are common in patients with SARS CoV-2. Wang et al. reported $16.7 \%$ (23/138) prevalence of cardiac arrhythmias in SARS CoV-2 out of which 16 (44.4\%) required ICU care. However, details of these arrhythmias were not mentioned. ${ }^{25} \mathrm{Hui}$ and colleagues reported ECG findings in 17 patients, of whom only three had tachycardia in severe and 
critical cases. ${ }^{48}$ Atrial fibrillation was reported in two patients with critical illness both of whom had a fatal outcome. Guo et al. reported ventricular tachycardia/ventricular fibrillation in $5.9 \%(11 / 187)$ patients with SARS-CoV-2 infection. ${ }^{35}$ Patients with elevated cardiac troponins had a significantly higher frequency of malignant arrhythmias compared to those without (9 vs $2 ; \mathrm{p}<0.001$ ) it. This highlighted the fact that patients with myocardial injury had far greater prevalence of tachyarrhythmias. This high frequency of arrhythmias may in part be due to metabolic causes such as electrolyte disturbances, neurohormonal activation or hypoxia especially in those who are critically ill. Similar findings have been documented in previous SARS pandemic with tachycardia being most common finding seen in $2 / 3$ rd of patients. ${ }^{49}$

\section{Venous Thromboembolism}

Patients with COVID-19 disease are at a high risk of Venous Thromboembolism (VTE) especially those who are critically ill with prolonged immobilization. Apart from venous stasis due to prolonged immobilization, hypercoagulability due to use of steroids, immunoglobulins as well as vascular endothelial damage due to central venous catheterization and/or ECMO can often contribute to the occurrence of VTE. Hypoxia too has been postulated as one of the causes of hypercoagulable states in these patients. Although none of the studies have reported prevalence of VTE, few case reports have mentioned the occurrence of pulmonary embolism in these patients. Xie et al reported acute pulmonary embolism in two patients aged 57 and 70 years with elevated D-dimer and multiple filling defects on CT pulmonary angiogram. ${ }^{50}$ Danzi et al reported a case of 75-year-old female with pulmonary embolism and right ventricular dysfunction on TTE. ${ }^{51}$ Multiple studies from China have reported higher D-dimer levels in SARS CoV2 patients with adverse outcomes. Zhou et al. reported elevated D-dimer levels which was strongly associated with greater in-hospital mortality (OR: 18.4; $p=0.003) .{ }^{52}$ Another study showed that $D$-dimer levels were significantly higher in non-survivors than survivors $(2.12 \mu \mathrm{g} / \mathrm{ml}$ vs $0.61 \mu \mathrm{g} / \mathrm{ml}$; $p<0.001$ ) thus reflecting a worse prognosis. Majority of the non-survivors met the criteria for DIC. ${ }^{53}$ Multiple reasons can be postulated for activation of coagulation cascade in critically ill-patients which include i) pro-inflammatory cytokines leading to activation of coagulation cascade especially in critically ill patients and D-dimer is a marker of fibrinolytic activity; ii) during inflammatory conditions, the alveolar hemostatic balance is tilted more towards a prothrombotic state; iii) proinflammatory cytokines may lead to endothelial injury and activation of coagulation cascade. ${ }^{54-56}$ Levels of D-dimer which serves as marker of fibrinolytic activity is elevated along with other inflammatory cytokines. Critically ill patients with SARS CoV-2 are at an increased risk for venous thrombosis and hence the need for anticoagulation in these patients. In the Chinese cohort of SARS CoV-2 patients, an early use of anticoagulation was initially recommended. ${ }^{57} \mathrm{D}$ dimer has a limited predictive value for venous thromboembolism especially in critically ill and hospitalized patients hence, VTE risk assessment should be done on an individualized basis. Another factor to be considered regarding anticoagulation is that most of the patients are elderly with multiple comorbid conditions hence an increased bleeding risk. A recent study showed that in COVID-19 positive patients with sepsis-induced coagulopathy score $<4$, administration of heparin led to reduced 28-day mortality. This showed that anticoagulant therapy had better outcomes in only selected group of patients. ${ }^{58}$ Anticoagulation with heparin thus has been recommended by few experts in China based on the limited data. ${ }^{59}$ Many authors suggested the use of Low Molecular Weight Heparin (LMWH) over Unfractionated Heparin (UFH) for the treatment of confirmed or suspected VTE whenever possible in patients with SARS CoV-2 infection. This approach avoids additional laboratory monitoring, minimizes nursing and phlebotomy exposure, and limits use of personal protective equipment. Due to lack of evidence on outcomes for bleeding or thrombosis, authors do not recommend dosing adjustments of LMWH using anti-Xa levels. They recommend use of UFH over LMWH in patients with acute kidney injury or in patients with creatinine clearance less than $15-30 \mathrm{ml} / \mathrm{min}$. They recommend using an anti-Xa assay rather than an aPTT to monitor therapeutic UFH in patients with COVID-19 whose aPTT is prolonged at baseline. If the baseline aPTT is normal, it is reasonable to monitor therapeutic UFH with either an anti-Xa assay or aPTT. They also suggested the clinicians to consider reasons other than SARS CoV-2 for baseline aPTT prolongation, as this laboratory finding could be due to an underlying coagulopathy that increases the risk of anticoagulant associated bleeding. ${ }^{60}$

\section{Cardiomyopathy and Heart Failure}

Several studies have noted the occurrence of cardiomyopathy in patients with SARS CoV-2. Among 21 critically ill patients with SARS CoV-2, cardiomyopathy developed in 7 (33.3\%) patients. ${ }^{61}$ Meanwhile, in a single-centered observational study of 187 patients with confirmed SARS CoV-2 eight patients had preexisting cardiomyopathy although little follow up evaluation was performed on the outcome in these patients. ${ }^{37,62}$ It is worth noting that a number of medications used in SARS CoV-2 may also lead to cardiomyopathy, including chloroquine, and interferon. ${ }^{63}$ Heart failure is a common complication of SARS CoV-2 due to deterioration of preexisting cardiac function and newly developed cardiomyopathy and myocarditis. In a multi-centered cohort study involving 191 SARS CoV-2 patients, heart failure was noted in $23 \%$ of patients, and more prevalent in non-survivors compared to survivors (52\% versus $12 \%$, 
$P<0.0001) .{ }^{64}$ Heart failure is characterized by decreased left ventricular ejection fraction and drastically elevated NT-proBNP. Guo and colleagues reported a higher level of cardiac biomarkers and NT-proBNP in patients with elevated troponin T. $^{33}$ Moreover, a tight correlation was identified between NT-proBNP and troponin T levels, indicating that patients with myocardial injury are at higher risks of cardiac dysfunction or heart failure. ${ }^{33}$ Although patients of SARS CoV-2 patients often have comorbidities that can affect cardiac diastolic function like diabetes, hypertension and obesity, few studies so far have revealed a relationship between Heart Failure with preserved Ejection Fraction (HFpEF) and SARS CoV-2. Sinkey and colleagues reported that HFpEF developed in a postpartum patient with SARS CoV-2 and preeclampsia. ${ }^{65}$ Notably, loss of AngiotensinConverting Enzyme 2 (ACE2), the receptor for SARS CoV-2, increases the proinflammatory macrophage phenotype in the heart from patients with HFpEF. ${ }^{66}$ More studies are warranted to explore the interplay between SARS CoV-2 and HFpEF. In SARS CoV-2 patients heart failure can be attributable to myocardial injury, systemic inflammatory response, ARDS and pulmonary hypertension, retention of water and sodium, renal dysfunction and imbalance between myocardial oxygen demand and supply.

\section{Cardiogenic Shock}

Although direct evidence for incidence rate of cardiogenic shock in patients infected with SARS CoV-2 is little, cardiogenic shock has been demonstrated as a severe complication of SARS CoV-2. In a 69-year-old patient with confirmed SARS CoV-2, elevated inflammatory markers and increased hypersensitive troponin I were noted prior to the development of severe cardiogenic shock. ${ }^{67}$ Cardiogenic shock may be mixed with other types of shock, like septic shock in SARS CoV-2 infection. In a study involving 138 patients with SARS CoV-2, $8.7 \%$ of patients had confirmed shock but subtypes of the shock were not mentioned. Shock was seen more often in patients who were admitted to ICU than in the non- ICU patients $(30.6 \%$ Vs $1.0 \%, P<0.001) .68$

\section{Management Related Cardiac Manifestation}

As the COVID-19 pandemic has widened its grip, "off label" re-purposing of various drugs such as $\mathrm{HCQ}$, azithromycin and lopinavir/ritonavir has been done in a bid to halt its march. However, with the usage of these drugs there has been the inadvertent risk of QT prolongation, TdP and sudden cardiac death. HCQ has shown promising results in in-vitro studies and is being increasingly used both for treatment as well as post-exposure chemoprophylaxis. ${ }^{69}$ However, it can prolong the QT interval and increase risk of TdP especially in patients with congenital long QT syndrome. This risk further increases with concomitant use of drugs like azithromycin or lopinavir and ritonavir with HCQs. A baseline 12-lead electrocardiogram should be performed in all SARS CoV-2 positive patients planned to receive these drugs along with serial monitoring in those with prolonged QTc interval. In addition, renal and hepatic functions, serum electrolytes should also be measured. In patients with QTc values <99th percentile for age/gender (460 $\mathrm{ms}$ in prepubertal males/females, $470 \mathrm{~ms}$ in postpubertal males, and $480 \mathrm{~ms}$ in postpubertal females) there is a low risk of $\mathrm{TdP}$ and hence drugs such as $\mathrm{HCQ} /$ chloroquine, lopinavir ritonavir or azithromycin can be initiated without delay. In patients with QTc $>500$ ms, a search should be made to identify all correctable cause of prolonged QT (drugs, electrolytes) and a risk benefit analysis should be done prior to start of therapy. If these patients are started on such drugs, it is recommended to use HCQ alone rather than a combination with azithromycin and frequently monitor the QTc intervals. In patients with QTc values $<99$ th percentile for age/gender prior to therapy and while on treatment, the QTC $>500$ ms or QTc increases by $>60$ $\mathrm{ms}$, azithromycin should be discontinued and or dose of hydroxychloroquine be reduced followed by daily ECG monitoring. If despite this the QTc $>500$ ms, it is prudent to perform a risk-benefit analysis in order to discontinue HCQ. ${ }^{58,69}$ In addition, chloroquine affects beta-receptor blockers through inhibition of CYP2D6. ${ }^{70}$ Therefore, blood pressure and heart rate must be closely monitored when co-administration of $\beta$-blockers and chloroquine in SARS CoV-2 patients. Remdesivir, previously administrated to patients with Ebola viral infection, is now being used in COVID-19 patients. During Ebola outbreak, one patient (among a total of 175 patients) administered with loading dose of remdesivir developed severe hypotension and sudden cardiac arrest. ${ }^{71}$

\section{COVID-I 9 with Underlying Cardiovascular Co-morbidities}

Patients with pre-existing co-morbidities tend to be more vulnerable to SARS CoV-2 infection as well as its complications with poor clinical outcomes. A meta-analysis of 8 studies including 46,248 patients from China reported that most common comorbidities in this population group were hypertension $(17 \pm 7 \%, 95 \% \mathrm{Cl}: 14-22 \%)$, diabetes

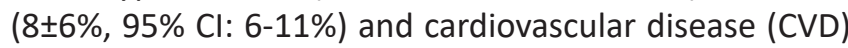
(5 $\pm 4 \%, 95 \% \mathrm{Cl}: 4-7 \%)$. The odds of hypertension (OR: 2.36 , 95\% Cl: 1.46-3.83) and CVDs (OR: 3.42, 95\% Cl: 1.88-6.22) were higher in severe patients as compared to non-severe group. ${ }^{72}$ In one of the largest series of SARS CoV-2 patients $(n=44,672)$, comorbidities such as hypertension were reported in $12.8 \%$, diabetes in $5.3 \%$ and CVD in $4.2 \%$ subjects. The case fatality rate (CFR) was higher among patients with co-morbidities such as CVD (10.5\%), diabetes (7.3\%) and hypertension (6\%) as compared to those without (CFR:0.9). ${ }^{3}$ another study among 1591 patients from Italy reported hypertension (49\%), CVDs (21\%) and hypercholesterolemia (18\%) in SARS CoV-2 patients. Patients 
with hypertension were significantly older, required higher PEEP levels, had lower $\mathrm{PaO} 2 / \mathrm{FiO} 2$ and higher ICU mortality. ${ }^{73}$ data regarding mortality in COVID-19 released by National Health Commission of China showed that $35 \%$ of patients were hypertensive while $17 \%$ had prior history of CAD. ${ }^{74}$ Similar findings were previously observed in SARS pandemic as well as MERS-CoV outbreak. ${ }^{75,76}$ In SARS outbreak, comorbidities such as diabetes and CVD were reported in $11 \%$ and $8 \%$ subjects respectively with an increased risk of mortality in these groups. ${ }^{74}$ Similarly, in MERS-CoV outbreak, diabetes and hypertension were present in nearly half of the cases while CVD in nearly one-third of them. ${ }^{77}$ The proposed hypothesis for increased severity of disease in patients with CVDs is that a majority of them are elderly with lower ACE2 levels and higher angiotensin signaling. As SARS-CoV-2 virus binds to ACE2, there occurs a decreased ACE2 expression and hence critically low ACE2 levels leading to higher angiotensin II levels. This leads to a more severe expression of disease in patients with co-morbidities. ${ }^{78}$

\section{Long Term Impact of Covid-19 on Cardiovascular System}

As we are still in the nascent stages of COVID-19 pandemic, data regarding the long-term impact on the cardiovascular system need to be evaluated. Sparse data exists regarding the long term impact of SARS CoV-2 infection on the cardiovascular system. Hospitalization for pneumonia increases both short term as well as long term CVD (myocardial infarction, stroke, and fatal coronary heart disease) risk. This has been attributed to the heightened systemic inflammatory and pro-coagulant activity seen in these patients. ${ }^{79}$ It has been seen that survivors of SARS epidemic have suffered metabolic derangements over a long follow-up period. A study involving 25 SARS survivors showed that these patients had higher predisposition to develop hyperlipidemia (68\%), CVA (44\%) and abnormal glucose metabolism (60\%) over a period of 12 years as compared to healthy volunteers. In addition, these patients had significantly higher lipid levels as compared to the controls which had been attributed to the high-dose pulses of methylprednisolone used during the acute illness. ${ }^{80}$ however, in a 12-year longitudinal study to determine long-term outcomes in patients with SARS treated with oseltamivir, no significant difference in cardiac parameters was reported among the two groups. ${ }^{81}$

Many patients developing ARDS survive the acute phase of the illness but a substantial proportion may die as a result of progressive pulmonary fibrosis. ${ }^{82}$ Importantly, an autopsy based study of 159 patients having ARDS, fibrosis was found to be in $4 \%$ of patients with disease duration of less than 1 week, (24\%) of patients with disease duration between 1-3 weeks, and (61\%) in patients with disease duration more than 3 weeks. This suggests that any potential antifibrotic treatment should be considered from the very first week of ARDS onset. ${ }^{83}$ A substantial proportion of patients developing ARDS will have residual long-term impairment of lung function and radiological (CT) evidence of pulmonary fibrosis with anterior reticulation, which is the dominant abnormality seen in upto $85 \%$ of survivors. ${ }^{82,84}$ Multiple aberrant host pathways interconnect resulting in pulmonary fibrosis in a subset of individuals developing ARDS. Dysregulated release of matrix metalloproteinases during the inflammatory phase of ARDS causes endothelial and epithelial injury, and unchecked fibro proliferation. In this regard, canonical profibrotic pathways regulated by TGF- $\beta 47$ are very important and vascular dysfunction being a key component for switching from ARDS to fibrosis, with VEGF97 and cytokines like TNF $\alpha$ and IL-6 implicated in it. ${ }^{82,85}$ It is not clear why certain individuals recover from such an insult whereas others show progressive pulmonary fibrosis. Data from previous coronavirus infections like SARS and MERS and upcoming data from current SARS CoV-2 pandemic suggests that substantial fibrotic consequences may there following SARS CoV-2 infection.

Chronic hypoxemia due to fibrosis and loss of lung parenchyma might contribute to the development of pulmonary hypertension and right ventricular hypertrophy by obliterating pulmonary vascular beds. ${ }^{86}$ Chronic hypoxemia may lead to myocardial oxygen demand supply mismatch resulting into myocardial damage as well. Antifibrotic therapy available so far theoretically might have a role in preventing fibrosis developing after SARS-CoV-2 infection but their exact role is yet to be seen.

\section{Conclusion}

The ACE2 receptors located in the lungs work as an attachment point for COVID-19 which leads to coronavirus disease (COVID-19)-related pneumonia. Since ACE2 is found in cardiac tissues also, it causes acute myocardial injury and chronic damage to the cardiovascular system. So, particular attention should be given to cardiovascular involvement during treatment for COVID-19 infection especially those who are critically ill. In addition, further research is needed detailing the epidemiology, exact pathophysiological mechanism, treatment as well as short- and long-term prognosis in these patients.

\section{Conflict of Interest: None}

\section{References}

1. Wu JT, Leung K, Leung G. Nowcasting and forecasting the potential domestic and international spread of the 2019-nCoV outbreak originating in Wuhan, China: a modelling study. Lancet. 2020; 395(10225): 689-697.

2. Lauer SA, Grantz KH, Bi Q et al. The incubation period of coronavirus disease 2019 (COVID-19) from publicly reported confirmed cases: estimation and application. 
Ann Intern Med 2020; 172(9): 577-582.

3. Wu Z, McGoogan JM. Characteristics of and important lessons from the coronavirus disease 2019 (COVID-19) outbreak in China: summary of a report of 72314 cases from the Chinese Center for Disease Control and Prevention. JAMA 2020; 323(13): 1239-1242.

4. Huang C, Wang $Y$, Li X et al. Clinical features of patients infected with 2019 novel coronavirus in Wuhan, China. Lancet 2020; 395(10223): 497-506.

5. Chen N, Zhou M, Dong X et al. Epidemiological and clinical characteristics of 99 cases of 2019 novel coronavirus pneumonia in Wuhan, China: a descriptive study. Lancet 2020; 395(10223): 507-513.

6. Jatin $\mathrm{M}$, Jonathan $\mathrm{H}$ et al. The Natural History, Pathobiology, and Clinical Manifestations of SARSCoV-2 Infections. J Neuroimmune Pharmacol 2020; 21: 1-28.

7. Turner, A, Hiscox, JA, Hooper NM. ACE2: from vasopeptidase to SARS virus receptor. Trends Pharmacol Sci 2004 Jun; 25(6): 291-294.

8. Ferrario CM, Chappell MC, Tallant EA. Counterregulatory actions of angiotensin-(1-7). Hypertension 1997; 30(3 Pt 2): 535-541.

9. Peiro S. Moncada S. Substituting angiotensin-(1-7) to prevent lung damage in SARS-CoV-2 infection? Circulation 2020; 141(21): 1665-1666.

10. Pedersen SF, Ho YC. SARS-CoV-2: a storm is raging. $J$ Clin Invest 2020; 130(5): 2202-2205.

11. Tisoncik JR, Korth MJ, Simmons CP. Into the eye of the cytokine storm. Microbiol. Mol Biol Rev 2012; 76 (1): 16-32.

12. Guo YR, Cao QD, Hong ZS et al. The origin, transmission and clinical therapies on coronavirus disease 2019 (COVID-19) outbreak - an update on the status. Mil Med Res 2020; 7(1): 11.

13. Kaplan B, Sulentic C, Haggerty H, Holsapple M. Toxic Responses of the Immune System. In Barnes and Davis ( $9^{\text {th }}$ Eds). Toxicology (Ch 12). New York: McGraw Hill Education.

14. Li B, Yang J, Zhao F et al. Prevalence and impact of cardiovascular metabolic diseases on COVID-19 in China. Clin Res Cardiol 2020; 109(5): 531-538.

15. Miller CC, Zarubaev VV et al. Influenza epidemics and acute respiratory disease activity are associated with a surge in autopsy-confirmed coronary heart disease death: results from 8 years of autopsies in 34,892 subjects. Eur Heart J 2007; 28(10): 1205-1210.

16. Nguyen JL, Yang W, Ito K, Matte TD, Shaman J, Kinney PL. Seasonal influenza infections and cardiovascular disease mortality. JAMA Cardiol 2016; 1(3): 274-281.

17. Smeeth L, Thomas SL, Hall AJ, Hubbard R, Farrington $P$, Vallance $P$. Risk of myocardial infarction and stroke after acute infection or vaccination. N Engl J Med 2004; 351(25): 2611-2618.

18. Bone RC, Grodzin CJ, Balk RA. Sepsis: a new hypothesis for pathogenesis of the disease process. Chest 1997; 112(1): 235-243.

19. Dong L,Hu S, Gao J, Discovering drugs to treat coronavirus disease 2019 (COVID- 19). Drug Discov Ther 2020; 14 (1): 58-60.

20. Mueck W, Kubitza D, Becka M, Co-administration of rivaroxaban with drugs that share its elimination pathways: pharmacokinetic effects in healthy subjects. Br J Clin Pharmacol 2013; 76(3): 455-466.

21. Yu CM, Wong RS, Wu EB, Kong SL, Wong J. Cardiovascular complications of severe acute respiratory syndrome. Postgrad Med 2006; 82(964): 140-144.

22. Sarkisian L, Saaby L, Poulsen TS, Gerke O, Jangaard N. Clinical characteristics and outcomes of patients with myocardial infarction, myocardial injury, and nonelevated troponins. Am J Med 2016; 129(4): 446446.

23. Huang C, Ren YL, Zhao J, Hu Y. Clinical features of patients infected with 2019 novel coronavirus in Wuhan, China. Lancet 2020; 395(10223): 497-506.

24. De Vries. SARS-cov-2/COVID-19: a primer for cardiologists. Neth Heart J 2020; 28(7-8): 366-383.

25. Wang D, Hu B, Hu C et al. Clinical characteristics of 138 hospitalized patients with 2019 Novel CoronavirusInfected Pneumonia in Wuhan, China. JAMA 2020; 323(11): 1061-1069.

26. Lippi G, Lavie CJ. Sanchis-Gomar F. Cardiac troponin I in patients with coronavirus disease 2019 (COVID-19): Evidence from a meta-analysis. Prog Cardiovasc Dis 2020; 63(3): 390-391.

27. Clerkin KJ, Fried JA, Raikhelkar J et al. Coronavirus disease 2019 (COVID-19) and cardiovascular disease. Circulation 2020; 141(20): 1648-1655.

28. Xu Z, Shi L, Wang Y et al. Pathological findings of COVID19 associated with acute respiratory distress syndrome. Lancet Respir Med 2020; 8: 420-422.

29. Tavazzi G, Pellegrini C, Maurelli M et al. Myocardial localization of coronavirus in COVID-19 cardiogenic shock. Eur J Heart Fail 2020; (22)5: 911-915.

30. Wong CK, Lam CWK, Wu AKL et al. Plasma inflammatory cytokines and chemokines in severe acute respiratory syndrome. Clin Exp Immunol 2004; 136: 95-103.

31. Saini HK, Xu YJ, Zhang M et al. Role of tumour necrosis factor- alpha and other cytokines in ischemiareperfusion-induced injury in the heart. Exp Clin Cardiol 2005; 10: 213-222.

32. He XW, Lai JS, Cheng J et al. Impact of complicated myocardial injury on the clinical outcome of severe or critically ill COVID-19 patients. Zhonghua Xin Xue Guan Bing Za Zhi 2020; 48(6): 456-460. 
33. Kwong JC, Schwartz KL, Campitelli MA et al. Acute myocardial infarction after laboratory-confirmed influenza infection. N Engl J Med 2018; 378(4): 345-353.

34. Shi S, Qin M, Shen B et al. Association of cardiac injury with mortality in hospitalized patients with COVID-19 in Wuhan, China. JAMA Cardiol 2020; 5(7): 802-810.

35. Guo T, Fan $\mathrm{Y}$, Chen $\mathrm{M}$, et al. Cardiovascular implications of fatal outcomes of patients with coronavirus disease 2019 (COVID-19). JAMA Cardiol 2020; 5(7): 811-818.

36. Arentz M, Yim E, Klaff L, Lokhandwala S, Riedo F, Chong $M$, Lee M. Characteristics and Outcomes of 21 Critically III Patients With COVID-19 in Washington State. JAMA 2020; 323(16): 1612-1614.

37. Madjid $M$ et al. Influenza epidemics and acute respiratory disease activity are associated with a surge in autopsy-confirmed coronary heart disease death: results from 8 years of autopsies in 34,892 subjects. Eur Heart J 2007; 28: 1205-1210.

38. 38. Bangalore $S$ et al. ST-segment elevation in patients with COVID-19 - a case series. N Engl J Med 2020; 382: 2478-2480.

39. Stefanini GG et al. ST-elevation myocardial infarction in patients with COVID-19: clinical and angiographic outcomes. Circulation 2020; 141: 2113-2116.

40. Libby P, Tabas I, Fredman G, Fisher EA. Inflammation and its resolution as determinants of acute coronary syndromes. Circ. Res. 2014 Jun;114:1867-1879.

41. Varga Z et al. Endothelial cell infection and endotheliitis in COVID-19. Lancet 2020; 395(10234): 1417-1418.

42. De Rosa $S$ et al. Reduction of hospitalizations for myocardial infarction in Italy in the COVID-19 era. Eur Heart J 2020; 41(22): 2083-2088.

43. Baldi E et al. Out-of-hospital cardiac arrest during the COVID-19 outbreak in Italy. N Engl J Med 2020; 383(5): 496-498.

44. Zeng JH, Liu YX, Yuan J et al. First case of COVID-19 infection with fulminant myocarditis complication: a case report and insights. Infection 2020; 1-5.

45. Hu H, Ma F, Wei X, Fang Y. Coronavirus fulminant myocarditis saved with glucocorticoid and human immunoglobulin. Eur Heart J 2020; 190.

46. Hua A, O'Gallagher K, Sado D, Byrne J. Life-threatening cardiac tamponade complicating myo-pericarditis in COVID-19. Eur Heart J 2020; 41(22): 2130.

47. Ruan QR, Yang K, Wang WX, Jiang LY, Song JX. Clinical predictors of mortality due to COVID-19 based on an analysis of data of 150 patients from Wuhan, China. Intensive Care Med 2020; 46(5): 846-848.

48. Hui $H$, Zhang $Y$, Yang $X$ et al. Clinical and radiographic features of cardiac injury in patients with 2019 novel coronavirus pneumonia. Cureus 2020; 12(5): e8021.

49. Yu CM, Wong RS, Wu EB et al. Cardiovascular complications of severe acute respiratory syndrome. Postgrad Med J 2006; 82(964): 140-144.

50. Xie Y, Wang X, Yang P, Zhang S. COVID-19 complicated by acute pulmonary embolism. Radiology: Cardiothoracic Imaging. 2020 Mar 16; 2(2): e200067

51. Danzi GB, Loffi M, Galeazzi G, Gherbesi E. Acute pulmonary embolism and COVID-19 pneumonia: a random association? Eur Heart J 2020; 41(19): 1858.

52. Zhou F, Yu T, Du R, et al. Clinical course and risk factors for mortality of adult inpatients with COVID-19 in Wuhan, China: a retrospective cohort study. Lancet 2020; 395(10229): 1054-1062.

53. Tang N, Li D, Wang X, Sun Z. Abnormal coagulation parameters are associated with poor prognosis in patients with novel coronavirus pneumonia. J Thromb Haemost 2020; 18(4): 844-847.

54. Shorr AF, Thomas SJ, Alkins SA, Fitzpatrick TM et al. Ddimer correlates with proinflammatory cytokine levels and outcomes in critically ill patients. Chest 2002; 121: 1262-1268.

55. Gunther A, Mosavi P, Heinemann S et al. Alveolar fibrin formation caused by enhanced procoagulant and depressed fibrinolytic capacities in severe pneumonia. Comparison with the acute respiratory distress syndrome. Am J Respir Crit Care Med 2000; 161(2 Pt 1): 454-462.

56. Bone RC, Grodzin CJ, Balk RA. Sepsis: a new hypothesis for pathogenesis of the disease process. Chest 1997; 112: 235-243.

57. Shanghai Clinical Treatment Expert Group for COVID-19. [Comprehensive treatment and management of coronavirus disease 2019: expert consensus statement from Shanghai]. Chin J Infect 2020; 1-10.

58. Tang N, Bai H, Chen X et al. Anticoagulant treatment is associated with decreased mortality in severe coronavirus disease 2019 patients with coagulopathy. J Thromb Haemost 2020; 18: 1094-1099.

59. Li T, Lu H, Zhang W. Clinical observation and management of COVID-19 patients. Emerg Microbes Infect 2020; $9(1): 687-690$.

60. Barnes GD, Burnet A, Allen A et al. Thromboembolism and anticoagulant therapy during the COVID-19 pandemic: interim clinical guidance from the anticoagulation forum. J Thromb Thrombolysis 2020; 50(1): 72-81.

61. Kwong JC et al. Acute myocardial infarction after laboratory-confirmed influenza infection. $N$ Engl J Med 2018; 378: 345-353.

62. Arentz M, Yim E, Klaff L et al. Characteristics and outcomes of 21 critically ill patients with COVID-19 in Washington State. JAMA 2020; 323(16): 1612-1614.

63. Driggin $\mathrm{E}$, Madhavan M, Bikdeli M, Chuich T, Laracy J, Bondi-Zoccai G. Cardiovascular considerationsfor 
patients, health care workers, and health systems during the coronavirus disease 2019 (COVID-19) pandemic. J Am Coll Cardiol 2020; 75(18): 2352-2371.

64. Zhou F, Yu T, Du R, Fan G, Liu Y, Liu Z et al. Clinical course and risk factors for mortality of adult inpatients with COVID-19 in Wuhan, China: a retrospective cohort study. Lancet 2020; 395(10229): 1054-1062.

65. Sinkey RG, Rajapreyar I, Robbins L, Dionne-Odom J, Pogwizd S. Heart failure with preserved ejection fraction in a postpartum patient with superimposed preeclampsia and COVID-19. AJP Rep 2020; 10(2): e165-e168

66. Patel V, Shah S, Verma S, Oudit G. Epicardial adipose tissue as a metabolic transducer: role in heart failure and coronary artery disease. Heart Fail Rev 2017; 22(6): 889-902.

67. Tavazzi G, Pellegrini C, Maurelli M, Belliato M, Sciutti F, Bottazzi A. Myocardial localization of coronavirus in COVID- 19 cardiogenic shock. Eur J Heart Fail 2020; 22(5): 911-915.

68. Dawei Wang, Bo Hu, Chang Hu, Fangfang Zhu, Xing Liu, Zhiyong Peng, Clinical characteristics of 138 hospitalized patients with 2019 novel coronavirusinfected pneumonia in Wuhan, China, JAMA 2020; 323(11): 1061.

69. Giudicessi JR, Noseworthy PA, Friedman PA, Ackerman MJ. Urgent guidance for navigating and circumventing the QTc prolonging and torsadogenic potential of possible pharmacotherapies for coronavirus disease 19 (COVID-19). Mayo Clin Proc 2020; 95(6): 1213-1221.

70. Somer M, Kallio J, Pesonen U, Pyykkö K et al. Influence of hydroxychloroquine on the bioavailability of oral metoprolol. Br J Clin Pharmacol 2000; 49(6): 549-555.

71. Mulangu S, Dodd L, Davey R, Mbaya O, Proschan M, P.W. Grp, P.C.S. Team, A. Randomized, Controlled trial of Ebola virus disease therapeutics. N Engl J Med 2019; 381(24): 2293-2303.

72. Yang J, Zheng Y, Gou X et al. Prevalence of comorbidities in the novel Wuhan coronavirus (COVID-19) infection: a systematic review and meta-analysis. Int J Infect Dis 2020; 94: 91-95.

73. Grasselli G, Zangrillo A, Zanella A et al. Baseline characteristics and outcomes of 1591 patients infected With SARS-CoV- 2 admitted to ICUs of the Lombardy Region, Italy. JAMA 2020; 323(16): 1574-1581.

74. Zheng Y, Ma Y, Zhang J, Xie X. COVID-19 and the cardiovascular system. Nat Rev Cardiol 2020; 17: 259260.

75. Chan JW, Ng CK, Chan YH et al. Short term outcome and risk factors for adverse clinical outcomes in adults with severe acute respiratory syndrome (SARS). Thorax 2003; 58: 686-689.

76. Badawi A, Ryoo SG. Prevalence of comorbidities in the Middle East respiratory syndrome coronavirus (MERS-CoV): a systematic review and meta-analysis. Int J Infect Dis 2016; 49: 129-133.

77. Fielding B. MERS-CoV: Understanding the Latest Human Coronavirus Threat. Viruses 2018; 10(2): 93.

78. Ghatrif M, Cingolani O, Lakatta EG. The dilemma of coronavirus disease 2019, aging, and cardiovascular disease: insights from cardiovascular aging science. JAMA Cardiol 2020; 5(7): 747-748.

79. Corrales-Medina VF, Alvarez KN, Weissfeld LA et al. Association between hospitalization for pneumonia and subsequent risk of cardiovascular disease. JAMA 2015; 313(3): 264-274.

80. Wu Q, Zhou L, Sun X et al. Altered lipid metabolism in recovered SARS patients twelve years after infection. Sci Rep 2017; 7(1): 9110.

81. Guo L, Han Y, Li J et al. Long-term outcomes in patients with severe acute respiratory syndrome treated with oseltamivir: a 12-year longitudinal study. Int J Clin Exp Med 2019; 12(10): 12464-12471.

82. Masclans JR, Roca O, Munoz X et al. Quality of life, pulmonary function, and tomographic scan abnormalities after ARDS. Chest 2011; 139(6): 13401346.

83. Thille AW, Esteban A, Fernandez-Segoviano P et al. Chronology of histological lesions in acute respiratory distress syndrome with diffuse alveolar damage: a prospective cohort study of clinical autopsies. Lancet Respir Med 2013; 1(5): 395-401.

84. Desai SR, Wells AU, Rubens MB, Evans TW, Hansell DM. Acute respiratory distress syndrome: $\mathrm{CT}$ abnormalities at long-term follow-up. Radiology 1999; 210(1): 29-35.

85. Burnham EL, Janssen WJ, Riches DW, Moss M, Downey GP. The fibroproliferative response in acute respiratory distress syndrome: mechanisms and clinical significance. Eur Respir J 2014; 43(1): 276-285.

86. Scott D. Collum, Javier Amione-Guerra, Ana S. CruzSolbes, Amara DiFrancesco Pulmonary Hypertension Associated with Idiopathic Pulmonary Fibrosis: Current and Future Perspectives. Can Respir J 2017; 12: 1-12. 